\title{
Redescripción y reubicación sistemática del trematodo Topsiturvitrema verticalia (Trematoda: Digenea) en una familia nueva
}

Lía I. Lunaschi

División Zoología Invertebrados. Museo de La Plata, FCNyM Paseo del Bosque S/Nº 1900 La Plata. Buenos Aires. Argentina; lunaschi@museo.fcnym.unlp.edu.ar

Recibido 24-III-2003. Corregido 19-X-2005. Aceptado 15-III-2006.

\begin{abstract}
Redescription and systematic reclassification of the trematode Topsiturvitrema verticalia (Trematoda: Digenea) in a new family. The trematode species Topsiturvitrema verticalia Vélez and Thatcher 1990 is redescribed based on two paratype samples and new material from the small intestine of the bat Myotis levis (Geoffroy), from Bahía Samborombón, Buenos Aires province, Argentina. This species is characterized by the presence of a "rhynchus", that is, an anterior sucker-like attachment organ, by the position of the mouth on the ventral surface (surrounded by the ventral sucker), by two blind digestive structures, by the presence of two false saccular caeca located anteriorly and behind the anterior attachment organ, by the presence of the genital pore in the marginal anterior position of the body, and by the mid-dorsal position of the excretory pore. T. verticalia was originally described as a member of the family Lecithodendriidae (Lühe 1901), however, the presence of "rhynchus" and the position of the mouth opening in the ventral sucker constitute morphological features that preclude its inclusion in this family. On account of this, it is considered necessary to create a new family for the genus Topsiturvitrema Vélez and Thatcher 1990, which is included in a new family, Topsiturvitrematidae fam. nov. Furthermore, the geographic range of the species is expanded from Colombia to Argentina and M. levis is added to the species’ host list. Rev. Biol. Trop. 54 (3): 1041-1045. Epub 2006 Sept. 29.
\end{abstract}

Key words: Topsiturvitrema verticalia, Digenea, Carollia perspicillata, Myotis levis, Chiroptera, Colombia, Argentina.

Vélez y Thatcher (1990) describieron un nuevo género de digéneo de la familia Lecithodendriidae que nominaron Topsiturvitrema. Este género se halla hasta el momento representado por una única especie, T. verticalia Vélez y Thatcher 1990, que se halló en Colombia parasitando el intestino delgado de Carollia perspicillata (L.).

En esta oportunidad y continuando con el estudio de los digéneos parásitos de murciélagos (Lunaschi 2002a, b), se redescribe a T. verticalia y se incluye al género Topsiturvitrema en una nueva familia, al tiempo que se amplía la distribución geográfica de la especie y el registro de sus hospedadores, ya que éste constituye el primer registro en Myotis levis (Geoffroy) de Argentina.

\section{MATERIALES Y MÉTODOS}

Se analizaron 15 ejemplares de $M$. levis, capturados con una red de niebla en la Bahía Samborombón, Buenos Aires, Argentina. Las vísceras fueron analizadas en el campo y se recolectó en el intestino de uno de ellos, dos especímenes del género Topsiturvitrema. Los digéneos, fueron fijados en formol al $4 \%$ 
caliente, conservados en alcohol $96^{\circ}$, coloreados sin comprimir con carmín clorhídrico diluido en alcohol $96^{\circ}$ (1-6), deshidratados en la serie alcohólica tradicional, clarificados con creosota y montados en bálsamo de Canadá entre dos cubreobjetos. Se analizaron además, dos ejemplares paratipo de $T$. verticalia depositados en el Harold W Manter Laboratory of Parasitology, University of Nebraska, Lincoln con el No HWML 31133. Las medidas, dadas en $\mu \mathrm{m}$, incluyen mínima, máxima y media, entre paréntesis. Los especímenes de Myotis levis fueron depositados en la Colección Helmintológica del Museo de La Plata, FCNyM, con el № 5065.

\section{RESULTADOS}

\section{Topsiturvitrema verticalia \\ Vélez y Thatcher 1990}

(Fig. 1-4, Cuadro 1)

Redescripción: Los datos morfométricos de los paratipos y del nuevo material están dados en el Cuadro 1. Cuerpo pequeño, piriforme, con la región anterior muy corta y aguzada y la región posterior globosa, muy conspicua. Tegumento íntegramente cubierto por delicadas espinas. Órgano de fijación anterior o rhynchus similar a una ventosa terminal poco profunda, más ancha que larga y abierta hacia la superficie dorsal. Ventosa ventral grande y poco profunda, en posición pre-ecuatorial. Boca abierta en la ventosa ventral y relacionada directamente con la faringe; esófago corto y ciegos cortos que alcanzan el borde posterior de los testículos. Poro genital, sobre el margen derecho o izquierdo del cuerpo, inmediatamente por debajo del órgano de fijación; atrio genital canalicular, sobre el borde posterior del órgano de fijación. Falsa bolsa del cirro grande y esférica, ubicada entre el órgano de fijación y la ventosa ventral; contiene a la vesícula seminal larga y plegada sobre sí misma, numerosas células prostáticas grandes, la pars prostática y el corto ducto eyaculador que se comunica directamente con la base del atrio genital.
Testículos simétricos, laterales a la ventosa ventral, pre-ecuatoriales, de contorno liso y alargados en el sentido del eje longitudinal del cuerpo. Ovario, mediano o submediano, próximo al extremo posterior, esférico o de contorno irregular y alargado en sentido transverso. Receptáculo seminal uterino; glándula de Mehlis anterior o lateral al ovario; canal de Laurer no observado. Vitelaria ventral y lateral, formada por folículos grandes y superpuestos total o parcialmente a los testículos, pudiendo extenderse en el espacio ovario-testicular. Útero ocupando todo el cuerpo; metratermo corto. Huevos grandes y operculados. Vesícula excretora no observada. Poro excretor mediano y dorsal.

\section{DISCUSIÓN}

Los ejemplares recolectados del intestino de $M$. levis se encontraron coparasitando con Ochoterenatrema labda Caballero 1943 y Suttonia talaveraensis Lunaschi 2002.

El análisis del material tipo de T. verticalia permitió concluir que los ejemplares recolectados del intestino de $M$. levis se corresponden morfológicamente con aquellos descritos para Colombia por Vélez y Thatcher (1990) a pesar de presentar una gran diferencia en la forma del cuerpo. Por la apariencia que presentan los ejemplares provenientes de $M$. levis, se concluye que los autores interpretaron erróneamente la forma del cuerpo de su material y el funcionamiento de algunas de sus estructuras debido al grado de retracción de los ejemplares que analizaron. En los ejemplares paratipo estudiados se observa que la hendidura ventral descrita, corresponde al sitio de retracción e invaginación del cuerpo ya que la ventosa ventral se halla en posición lateral (Fig. 1). El nuevo material (Fig. 3) muestra que en vida esta especie no presenta el cuerpo dividido en dos hemisferios por una hendidura ventral como fue descrito, sino que es globoso por el gran desarrollo del útero y presenta una pequeña proyección anterior donde se ubica el órgano adhesivo (= acetábulo de Vélez y Thatcher 1990); en estas condiciones, la 
CUADRO 1

Morfometría de Topsiturvitrema verticalia Vélez y Thatcher 1990

TABLE 1

Morphometry of Topsiturvitrema verticalia Vélez y Thatcher 1990

Procedencia

Hospedero
Largo
Ancho
Rhynchus (rh) largo
ancho
Ventosa ventral (vv) largo
ancho
Relación ancho rh/vv
Faringe largo
ancho
Atrio genital largo
Falsa bolsa del cirro largo
(fbc) ancho
Relación ancho vv/fbc
Ovario largo
ancho
Testículo izquierdo largo
ancho
Testículo derecho largo
ancho
Huevos

Puerto Triunfo, Colombia

$$
\begin{gathered}
\text { Carollia perspicillata } \\
300-350(320) \\
290-430(392) \\
68-87(77) \\
87-112(98) \\
107-125(121) \\
137-155(149)
\end{gathered}
$$$$
100
$$$$
37
$$$$
62-87(72)
$$$$
75-85 \text { (78) }
$$$$
25-27 \times 9-12(26 \times 9)
$$

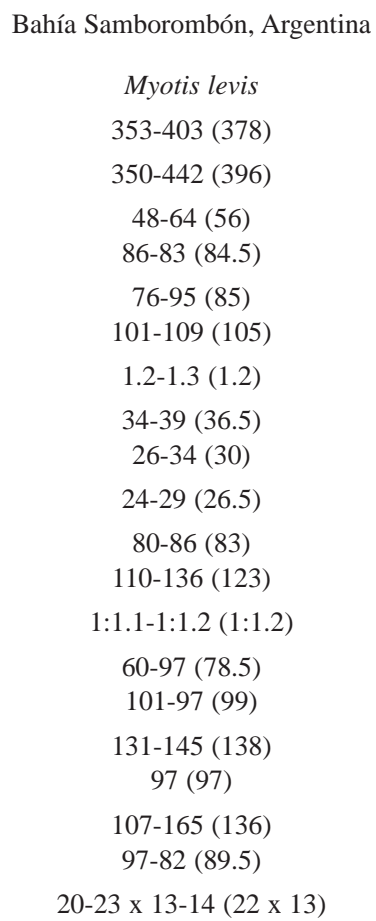

Medidas en $\mu$ m; mínima y máxima; media entre paréntesis.

ventosa ventral, donde se abre la boca (= ventosa oral de Vélez y Thatcher 1990), adopta una posición mediana, ventral y pre-ecuatorial (= subterminal según Vélez y Thatcher 1990), la faringe se dispone con el eje mayor hacia el órgano adhesivo y los ciegos se dirigen hacia la extremidad posterior. Del mismo modo, la genitalia terminal ha sido descrita conformada por un saco del cirro curvo, lateral al acetábulo y conteniendo al cirro y a la vesícula seminal. En el nuevo material no se ha observado la presencia de un cirro y la estructura descrita como tal resulta de la evaginación parcial del atrio genital, tal como se observa en uno de los ejemplares paratipo (Fig. 2, 4). La diferencia que se advierte entre los ejemplares paratipo y los de $M$. levis, se halla en la ubicación del poro genital. En el lote de C. perspicillata se halla aparentemente ubicado sobre el margen izquierdo del cuerpo (Fig. 2), y en los de $M$. levis, sobre el derecho (Fig. 4). Probablemente éste sea un carácter específico determinante para la creación de una nueva especie, pero se prefiere considerar a los especímenes de $M$. levis como representantes de $T$. verticalia hasta tanto se estudien nuevos ejemplares obtenidos del hospedador y la localidad tipo.

Finalmente, y a pesar de que Vélez y Thatcher (1990) argumentan la creación del género Topsiturvitrema y su inclusión en la familia Lecithodendriidae sólo por la orientación del cuerpo (Sic), se considera que las características anatómicas, que aportan los nuevos ejemplares, imposibilitan su inclusión en esta familia. La presencia de una ventosa ventral, de la boca desembocando en esta 

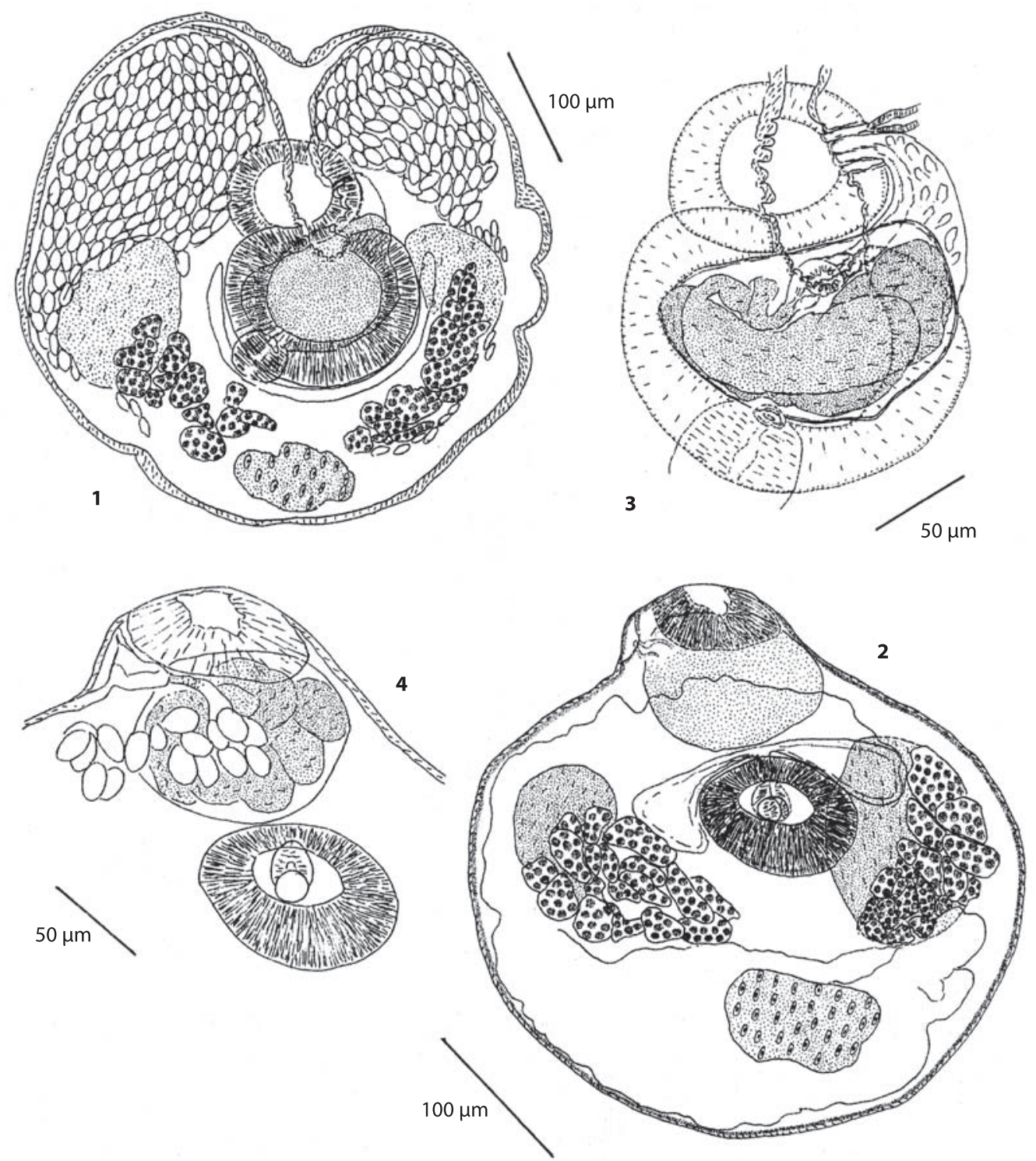

Fig. 1-2. Topsiturvitrema verticalia de Carollia perspicillata. Fig. 1. Paratipo, vista ventral. Fig. 2. Detalle de la falsa bolsa del cirro, vista ventral. Fig. 3-4. T. verticalia de Myotis levi. Fig. 3. Ejemplar in toto, vista ventral. Fig. 4. Detalle de la falsa bolsa del cirro, vista ventral. Abreviaciones: ag: atrio genital; b: boca; f: faringe; fbc: falsa bolsa del cirro; h: huevo; m: metratermo; pg: poro genital; rh: rhynchus; vs: vesícula seminal; vv: ventosa ventral.

Fig. 1-2. Topsiturvitrema verticalia from Carollia perspicillata. Fig. 1. Paratype from Carollia perspicillata, ventral view. Fig. 2. Enlarged ventral view of false cirrus pouch. Fig. 3-4. T. verticalia from Myotis levis. Fig. 3. Entire worm, ventral view. Fig. 4. Enlarged ventral view of false cirrus pouch. Abbreviations: ag: genital atrium; b: mouth; f: pharynx; fbc: false cirrus pouch; h: egg; m: metraterm; pg: genital pore; rh: rhynchus; vs: seminal vesicle; vv: ventral sucker. 
estructura, la presencia de dos ciegos digestivos, de una genitalia terminal conformada por una falsa bolsa del cirro ubicada en la extremidad anterior y del poro genital marginal, impide también su inclusión en la familia Bucephalidae Poche 1907, especialmente representada por parásitos de peces y excepcionalmente, reptiles (Yamaguti 1971). Por estos motivos, se propone la inclusión del género Topsiturvitrema en una nueva familia, Topsiturvitrematidae fam. nov.

\section{Topsiturvitrematidae fam. nov.}

Diagnosis: Cuerpo pequeño, transversalmente alargado, piriforme; tegumento íntegramente espinoso. Extremidad anterior con un órgano de fijación, o rhynchus, muscular y a modo de ventosa. Ventosa ventral débilmente desarrollada en la mitad anterior del cuerpo. Boca ubicada en la ventosa ventral. Prefaringe ausente. Faringe bien desarrollada. Esófago corto. Intestino con dos ramas ciegas cortas. Poro genital marginal y ubicado en la región anterior del cuerpo. Atrio genital canalicular. Falsa bolsa del cirro esférica, muy conspicua, ubicada en la región anterior del cuerpo; encierra vesícula seminal interna, pars prostática y numerosas células prostáticas. Testículos ecuatoriales, simétricos y laterales a la ventosa ventral. Ovario postesticular, mediano o submediano. Vitelaria folicular y lateral, en la mitad posterior del cuerpo. Receptáculo seminal uterino. Canal de Laurer aparentemente ausente. Útero ocupando todo el cuerpo. Huevos operculados. Vesícula excretora no observada. Poro excretor en posición mediana y dorsal. Parásitos del intestino de quirópteros.

\section{AGRADECIMIENTOS}

Agradecemos a Juliana Notarnicola por la sesión del material utilizado en esta investigación. A Gerardo Pérez Ponce de León por la lectura crítica del manuscrito. A Mariano Merino, encargado de la colección de la Sección de Mastozoología del Museo de Ciencias Naturales de la Universidad Nacional de La Plata, por la determinación taxonómica de los hospedadores. A Skip Sterner, Collection Manager y Scott L. Gardner, Curator of Parasitology, del Harold W. Manter Laboratory of Parasitology, University of Nebraska, Lincoln, Nebraska, EEUU, por el envío de los paratipos.

\section{RESUMEN}

Se redescribe el trematodo Topsiturvitrema verticalia Vélez y Thatcher 1990 con base en dos paratipos y material nuevo del intestino delgado del murciélago Myotis levis (Geoffroy), de Bahía Samborombón, Buenos Aires, Argentina. Esta especie es caracterizada por la presencia de un "rhynchus" u órgano de fijación anterior parecido a una ventosa terminal, la boca abierta en la superficie ventral (rodeada por la ventosa ventral), dos ciegos cortos, dos falsas bolsas del cirro localizadas anteriormente y detrás del órgano de fijación anterior, un poro genital sobre el margen anterior del cuerpo y el poro excretorio en posición dorsal. T. verticalia fue descrito como un miembro de la familia Lecithodendriidae (Lühe 1901); sin embargo, la presencia de "rhynchus" y la posición de la boca abierta en la ventosa ventral constituyen características morfológicas que imposibilitan su inclusión en esta familia. El género Topsiturvitrema Vélez y Thatcher 1990 se incluye en una familia nueva, Topsiturvitrematidae fam. nov., se amplía el ámbito geográfico de Colombia a Argentina y se agrega $M$. levis a la lista de hospederos de la especie.

Palabras clave: Topsiturvitrema verticalia, digenea, Carollia perspicillata, Myotis levis, Chiroptera, Colombia, Argentina.

\section{REFERENCIAS}

Lunaschi, L. 2002a. Tremátodos Lecithodendriidae y Anenterotrematidae de Argentina, México y Brasil. An. Inst. Biol. UNAM. 73: 1-10.

Lunaschi, L. 2002b. Redescripción y comentarios taxonómicos sobre Ochoterenatrema labda Caballero, 1943 (Digenea: Lecithodendriidae), parásito de quirópteros en México. An. Inst. Biol. UNAM. 73: 11-18.

Vélez, I. \& V.E. Thatcher. 1990. Cinco especies de Lecithodendriidae (Trematoda) en murciélagos de Colombia, incluyendo tres nuevos géneros. Rev. Bra. Zool. 7: 155-164.

Yamaguti, S. 1971. Synopsis of digenetic Trematodes of Vertebrates. Keigaku. Japon. Vol. I: 1074 p. Vol II: $349 \mathrm{pl}$. 
